\title{
Complications in MS Patients after CCSVI Procedures Abroad (Calgary, AB)
}

\author{
Jodie M. Burton, Katayoun Alikhani, Mayank Goyal, Fiona Costello, \\ Chris White, David Patry, Robert Bell, Michael D. Hill
}

\begin{abstract}
Background: The "chronic cerebrospinal venous insufficiency" or "CCSVI" hypothesis, namely that multiple sclerosis (MS) is caused by abnormalities in the azygous and internal jugular veins with subsequent alterations in venous hemodynamics in the central nervous system, has been a dominant topic in MS care in Canada over the past year. Although there is no methodologically rigorous evidence to support this hypothesis presently, a considerable number of MS patients have undergone endovascular CCSVI procedures. Such procedures include angioplasty or stent placement in jugular and azygous veins. The safety and efficacy of these procedures is unknown, but not without risk. Methods: Chart and patient review of five patients with confirmed MS followed in Calgary were undertaken after patients came to medical attention by referral or admission secondary to complications believed to be associated with CCSVI procedures. Results: Complications upon investigation and review included internal jugular vein stent thrombosis, cerebral sinovenous thrombosis, stent migration, cranial nerve injury and injury associated with venous catheterization. Conclusions: As the debate about CCSVI and its relationship to MS continues, the complications and risks associated with venous stenting and angioplasty in jugular and azygous veins are becoming clearer. As increasing numbers of MS patients are seeking such procedures, these five cases represent the beginning of a wave of complications for which standardized care guidelines do not exist. Our experience and that of our colleagues will be used to develop guidelines and strategies to monitor and manage these patients as their numbers increase.
\end{abstract}

RÉSUMÉ: Complications chez des patients atteints de SP après des interventions IVCC subies à l'étranger (Calgary, AB). Contexte : L'hypothèse de l'insuffisance veineuse céphalorachidienne chronique (IVCC), selon laquelle la sclérose en plaques (SP) est causée par des anomalies des veines azygos et jugulaire interne entraînant des altérations hémodynamiques veineuses dans le système nerveux central a dominé l'actualité concernant le traitement de la SP au Canada au cours de la dernière année. Bien qu'il n'y ait pas de données rigoureuses au point de vue méthodologique en faveur de cette hypothèse actuellement, un grand nombre de patients atteints de SP ont subi des interventions endovasculaires pour traiter 1'IVCC. Ces interventions comportent une angioplastie ou la mise en place d'une endoprothèse dans les veines jugulaires et azygos. La sécurité et l'efficacité de ces interventions ne sont pas connues et elles ne sont pas sans risques. Méthode : Nous avons révisé les dossiers de 5 patients suivis à Calgary pour SP confirmée et nous les avons examinés lorsqu'ils ont été référés ou ont été admis à l'hôpital pour des complications possiblement reliées aux interventions pour IVCC. Résultats : Suite aux examens pratiqués et à la revue des dossiers, les complications suivantes ont été notées : thrombose de l'endoprothèse de la veine jugulaire, thrombose sino-veineuse cérébrale, migration de l'endoprothèse, lésion d'un nerf crânien et lésions associées à la cathétérisation veineuse. Conclusions : Alors que le débat sur l'IVCC et sa relation à la SP continue, les complications et les risques associés à la mise en place d'une endoprothèse et à l'angioplastie des veines jugulaires et azygos se précisent. Du fait qu'un nombre croissant de patients atteints de SP ont recours à ces interventions, ces 5 cas représentent le début d'une vague de complications pour lesquelles il n'existe pas de lignes directrices standardisées de traitement. Notre expérience et celle de nos collègues sera utilisée pour élaborer des lignes directrices et des stratégies pour surveiller et traiter ces patients à mesure que leur nombre augmente.

Can. J. Neurol. Sci. 2011; 38: 741-746

Over the past 12 months, the theory of "chronic cerebrospinal venous insufficiency" or "CCSVI" as a potential cause of multiple sclerosis (MS) has been a dominant topic in MS care practices and research in Canada and abroad ${ }^{1,2}$. The CCSVI hypothesis proposes that MS patients have abnormal venous hemodynamics affecting the brain and spinal cord caused by structural abnormalities in the azygous and internal jugular (IJ) veins $^{1,2}$. Supporters of the CCSVI theory have suggested that venous distension may cause iron-laden erythrocytes to leak into surrounding tissues, provoking a secondary inflammatory cascade in the central nervous system $(\mathrm{CNS})^{1,2}$. However, subsequent methodologically more rigorous studies examining cranial venous drainage in MS patients and controls by multiple imaging modalities, including ultrasonography and magnetic resonance imaging (MRI), have not found an association between $\mathrm{MS}$ and anomalous or pathological venous flow ${ }^{3-8}$.

From the Department of Clinical Neurosciences (JMB, KA, FC, CW, DP, RB, MDH) Hotchkiss Brain Institute (JMB, MG, FC, CW, DP, RB, MDH), Department of Radiology (MG), Department of Community Health Sciences (MDH), University of Calgary, Calgary, Alberta, Canada.

Received February 23, 2011. Final Revisions Submitted March 18, 2011. Correspondence to: Jodie M. Burton, Health Sciences Centre, Room 1007, 3330 Hospital Drive NW, Calgary, Alberta, T2N 4N1, Canada. 
Despite the absence of evidence from methodologically rigorous studies validating this entity and its association with MS, a significant number of MS patients have sought endovascular treatment abroad as such procedures are not approved in Canada.

Common CCSVI endovascular procedures include angioplasty and/or stent placement in IJ and/or azygous veins. Local follow-up by the treating physician is not typically provided because the vast majority of patients are from abroad and return to their country of origin within days of the procedure. Postprocedure medical recommendations are highly varied, but often include brief, typically subcutaneous, anticoagulation followed by a longer course of antiplatelet or rarely, anticoagulant medications.

The safety and efficacy of angioplasty or stent placement for presumed venous stenosis of $\mathrm{IJ}$ and azygous veins is unknown. It remains unclear if the procedure is being applied to those with true and acceptable variability of the venous system or to those with pathological venous anatomy. Occlusion of the jugular veins does not appear to cause MS. Head and neck surgeons have occluded the jugular veins during radical neck surgery to treat cancer without seeing significant hemodynamic changes in the intracranial venous system ${ }^{9}$. Even with bilateral jugular ligation, intracranial venous integrity is maintained ${ }^{9}$. No case reports of demyelinating-like lesions or clinical events following such procedures exist in the literature ${ }^{9}$.

Previous CCSVI trials have reported adverse events, but in certain cases, this information has been limited in detail (i.e. timing, duration, rates and severity). In his 2009 trial publication, Zamboni et al reported that no operative or postoperative complications occurred in his trial, while at the same time reporting that bleeding from vascular access sites occurred "occasionally", and with acute post-procedure monitoring of only four hours". Ludyga et al also reported relatively minor complications with virtually no clinical consequences in over 344 endovascular interventions in 192 patients $^{10}$. In this trial, there were in fact two cases of stent thrombosis (considered by the author to be of little clinical significance given that these veins were previously "not patent"); five cases requiring hospitalization - up to one week for one gastrointestinal hemorrhage and typically one day for four relatively mild cases of venous access site bleeding; two cases of transient periprocedural atrial fibrillation requiring pharmacological intervention; four cases of stent migration requiring the placement of an additional stent; pseudoaneurysm development and surgical intervention to remove an angioplastic balloon ${ }^{10}$. Several of the aforementioned complications would be appropriately labelled serious adverse events. The most alarming adverse events that have been associated with CCSVI procedures, in these cases, stent placement, occurred at Stanford University in California in $2009^{11}$. These severe events included stent migration into the right cardiac ventricle requiring open heart surgery and a fatal brainstem hemorrhage"l.

The actual number of MS patients who have sought treatment for CCSVI outside of Canada is not known as many patients may not notify their local physicians. In Calgary, we are seeing increasing numbers of patients with MS returning from abroad having undergone venous procedures, and an increasing number of presumed associated complications. We report five of the initial cases of presumed CCSVI procedure associated complications, both symptomatic and asymptomatic.

\section{METHODS}

In this case series, approved by the Conjoint Health Ethics Research Board at the University of Calgary, we report the complications experienced by five patients with confirmed MS followed in Calgary after venous stenting and/or angioplasty procedures performed for presumed CCSVI outside of Canada. These cases were not collected under any a priori research protocol, but came to the attention of their care providers upon detection of complications, typically through emergency room visits. We are unable to comment in detail on the exact procedures or protocols employed by these centres because it is not clear standardized protocols are employed across these centres, and the content of documentation provided to patients upon discharge has proven to be highly variable and limited in detail.

\section{Results/Cases \\ Patient 1}

The first patient is a 40 year-old woman with a nine month history of relapsing-remitting MS (and a total of three relapses). Her most recent neurological assessment in 2010 , prior to her venous procedure, demonstrated no neurological deficits, and thus her Expanded Disability Status Scale (EDSS) score was 0 (consistent with no signs of neurological dysfunction). She had never used an MS disease modifying therapy. In August 2010, the patient travelled to Eastern Europe where a unilateral IJ vein stent (SMART 12x60mm) was placed for presumed CCSVI. She was discharged on acetylsalicylic acid (ASA) and

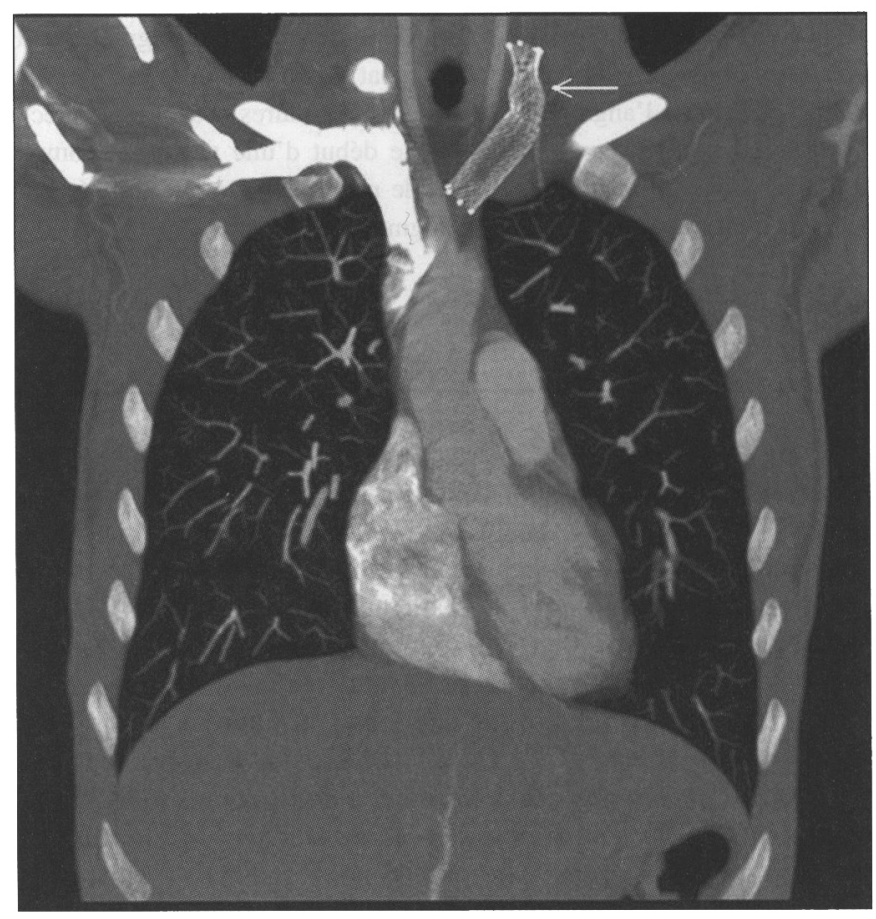

Figure 1: Complete occlusion of left internal jugular vein stent by thrombosis (see arrow) on CT venography (coronal view). 


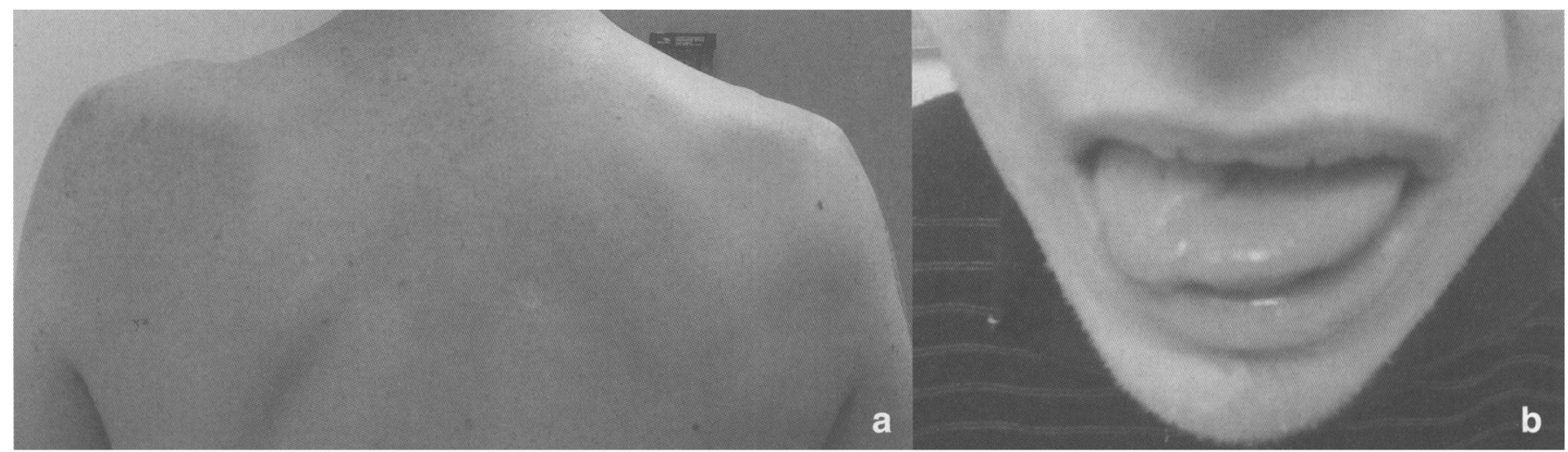

Figure 2: a) Left trapezius atrophy with scapular winging from presumed accessory nerve damage. b) Tongue deviation to the right with right sidedatrophy from presumed right hypoglossal nerve damage.

clopidogrel (75 mg/d) for six months. In October 2010 , during a follow-up screening ultrasound of the IJ veins at a private facility, an asymptomatic complete occlusion of the stent with presumed thrombus and possible aneurysmal dilatation of the stented IJ vein was detected. The patient was sent to the emergency department at Foothills Medical Centre in Calgary and admitted to the Stroke service for further investigation and treatment. A repeat jugular venous ultrasound was performed as well as a computed tomogram (CT) venogram. The CT venogram revealed that the IJ vein stent was completely occluded just below the skull base (Figure 1). Given that the patient was asymptomatic, she was discharged home with ongoing follow-up through the MS clinic and Stroke service, and advised to remain on her current dose of ASA and clopidogrel. The patient returned to the emergency department at Foothills Medical Centre two months later with a four day history of left- sided neck pain and a one day history of episodic sharp pleuritic chest pain. Her exam was normal with the exception of mild tachypnea. Chest and upper extremity CT angiographic imaging ruled out the presence of further venous thrombosis and pulmonary thromboembolism. She was discharged back into the care of her neurologist with previously arranged follow-up four weeks later.

\section{Patient 2}

A 26 year-old man with a two year history of relapsingremitting MS on interferon beta- $1 \mathrm{~b}$, with a baseline EDSS of 2.0 (indicating minimal neurological findings on examination), travelled to India in September 2010 where bilateral IJ vein stents were placed for presumed CCSVI. He was briefly treated with anticoagulation post-procedure, but the particular agent was

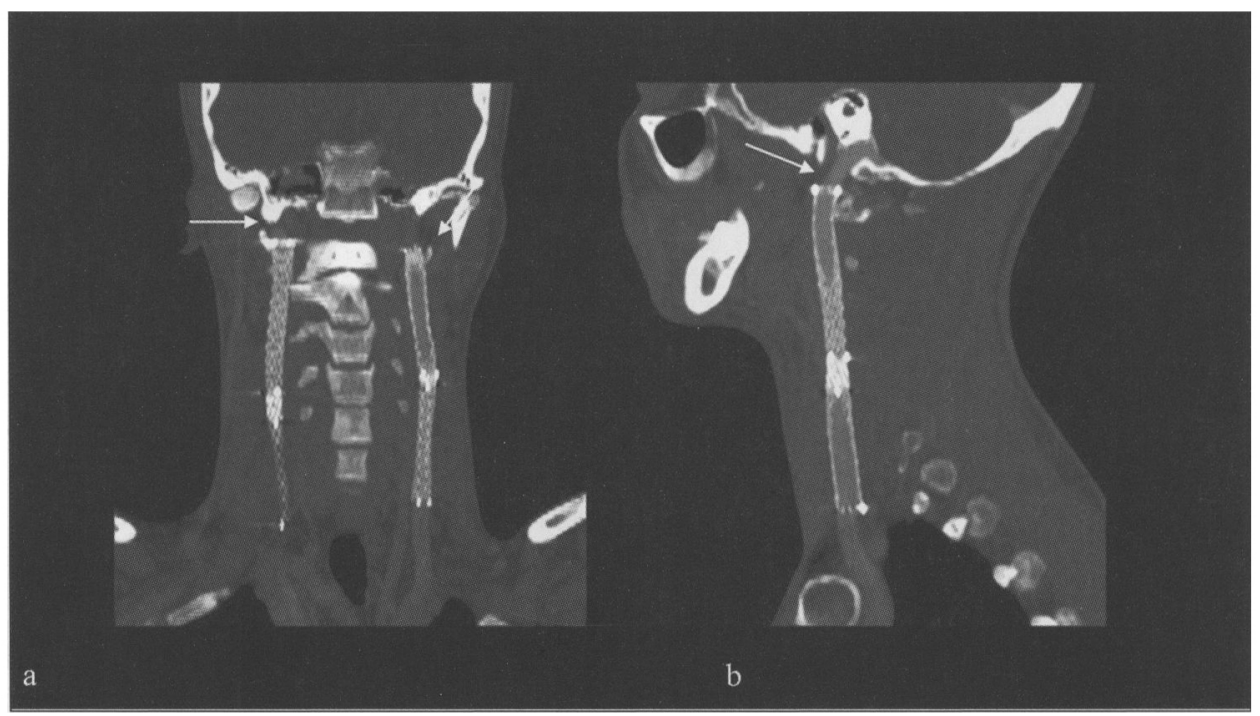

Figure 3: a) Coronal and (b) right sagittal CT venogram demonstrating extent of bilateral internal jugular vein stents and "transition zone" (see arrows) in which the cranial most part of the stent at CI$C 2$ level has resulted in stretching of the XI and XII nerves. 
not reported. He was discharged on ASA and clopidogrel (75 $\mathrm{mg}$ /day). Immediately following the procedure, the patient experienced the acute onset of cervical pain, dysarthria and dysphagia. Upon his return to Canada, an urgently arranged brain MRI showed no evidence of stroke or hemorrhage. Clinical reassessment at the University of Calgary MS Clinic six weeks later revealed new changes including considerable cervical pain elicited upon neck and shoulder muscle strength testing and prominent wasting of the left trapezius and sternocleidomastoid muscles (Figure 2a). He also had deviation of the protruded tongue to the right side, with atrophy but without fasciculations (Figure 2b). His EDSS score at this time was 2.5, indicating an increase in neurological deficits when compared to his preprocedure status. A subsequent electromyogram (EMG) demonstrated a right cranial nerve XI and XII partial axonotmesis and a left cranial nerve XI complete injury (versus combined axonotmesis) with ongoing conduction block. A CT venogram revealed bilateral oversized IJ stents that resulted in stretching of the hypoglossal and accessory nerves (Figure 3a and $b$ ).

\section{Patient 3}

A 39 year-old man with a ten year history of MS (having converted from relapsing-remitting to secondary progressive MS over six years ago), had a baseline EDSS of 6.5, indicating severe neurological deficits. Specifically, he had triplegia and an inability to ambulate more than 20-30 meters with a walker. The patient had bilateral jugular angioplasty in Eastern Europe in July 2010 for presumed CCSVI. Upon telephone assessment by the treating centre the following month, the patient reported that a brief improvement in overall energy in the early days following his angioplasty had abated, and he was told to return for azygous

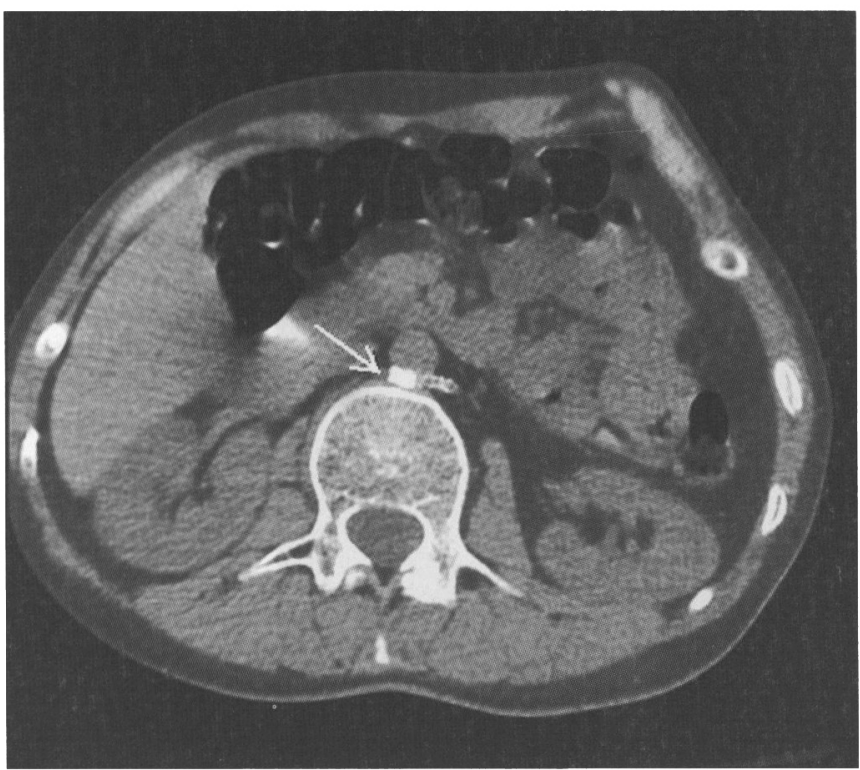

Figure 4: Axial CT scan of abdomen demonstrating venous stent initially stated to be placed in the azygous vein now visualized to in the left renal or paralumbar vein (see arrow). vein intervention. Although he did not provide consent for stent placement, a single azygous vein stent roughly $6 \mathrm{~cm}$ in length was placed in August 2010, and the patient was discharged on a six month course of ASA. Two months later, the patient was taken to his local emergency room with a single episode of uncomplicated syncope. While the syncope was felt to be unremarkable, routine chest and abdominal imaging suggested that his stent, presumably accurately placed in the azygous vein, had migrated. A CT of the chest and abdomen demonstrated that the stent was now most likely in his left renal vein or less likely, a paralumbar vein (Figure 4).

\section{Patient 4}

A 48 year-old woman with a 19 year history of MS, having converted to secondary progressive MS several years ago, had a baseline EDSS of 2.5, indicating minimal neurological deficits. She travelled to Mexico in July 2010 for bilateral jugular angioplasty for presumed CCSVI. The withdrawal of the balloon required surgical dissection of the femoral vein leading to femoral vein puncture and an extraperitoneal hematoma. The patient was treated with a red blood cell transfusion and a course of oral antibiotics. One month later, the patient was sent to the emergency department after an outpatient abdominal ultrasound for persistent groin pain since her procedure revealed a persistent extraperitoneal abdominal hematoma. The ultrasound and CT of the abdomen repeated in hospital revealed a large, complex, heterogeneous fluid collection representing a contained extraperitoneal hematoma (measuring 9.3 by 7.5 by $7.1 \mathrm{~cm}$ ) within the space of Retzius (anterior to the bladder) causing mild compression of the bladder (Figure 5). Conservative and expectant management was advised by General Surgery and Urology services.

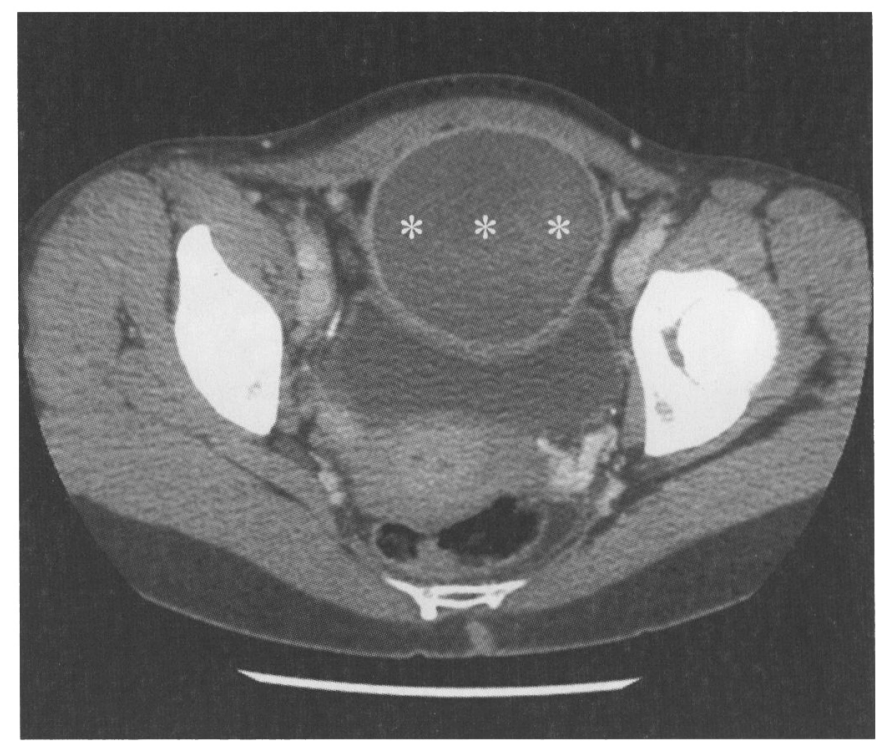

Figure 5: Axial view of $C T$ abdomen demonstrating extraperitoneal hematoma (measuring 9.3 by 7.5 by $7.1 \mathrm{~cm}$ ) in the space of Retzius anterior to the bladder exerting mass effect on the anterior aspect of the bladder (see asterixes). 

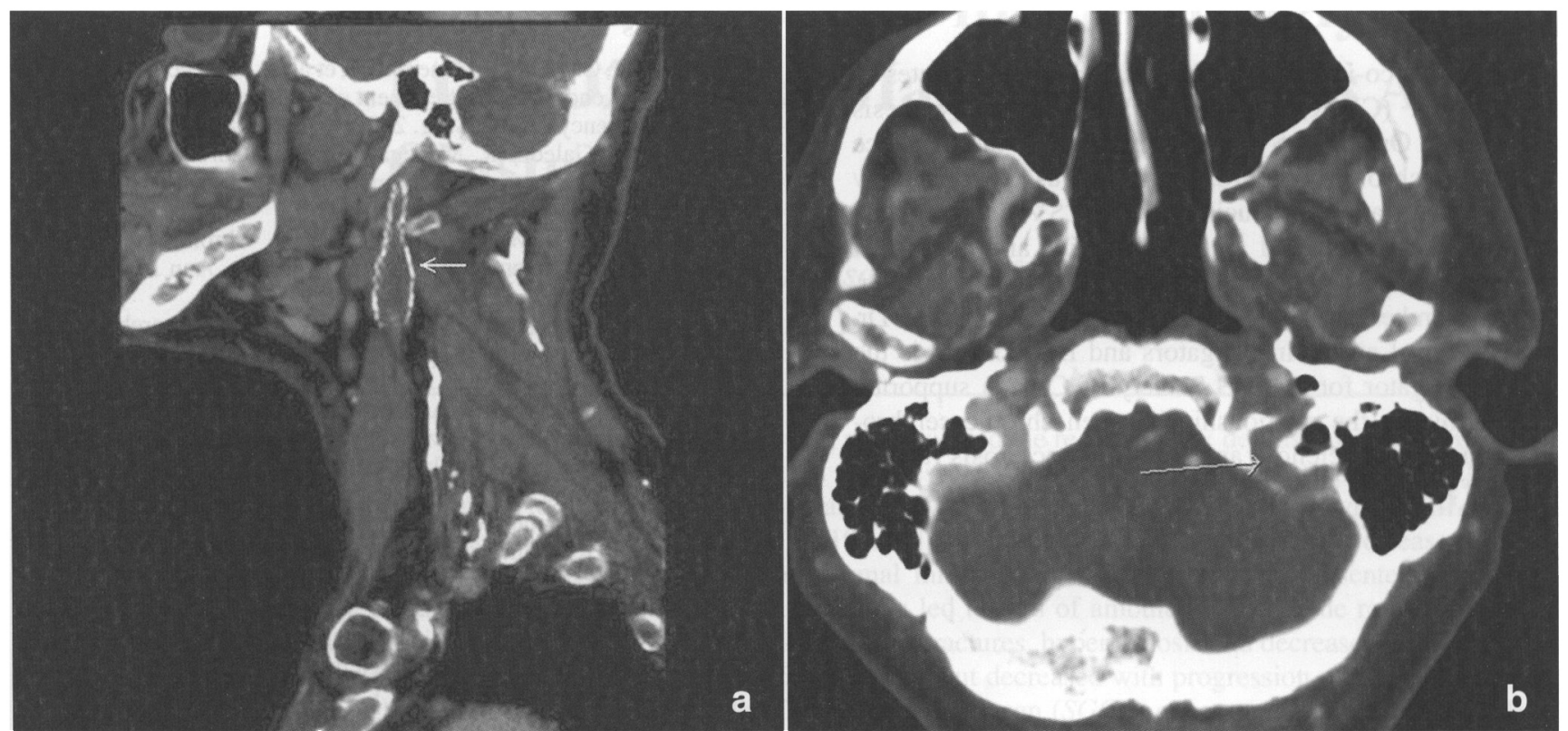

Figure 6: a) Sagittal CT venogram demonstrating complete thrombotic occlusion of left internal jugular vein stent (see arrow). (b) Axial CT venogram at the level of distal sigmoid sinus and jugular bulb shows absence of contrast opacification on the left side, possibly due to presence of thrombus extending from left internal jugular vein stent thrombus or slow flow.

\section{Patient 5}

The final patient is a 66 year-old man with a 20 year history of MS, having transitioned to secondary progressive MS several years earlier, and a baseline EDSS of 6.5 , indicating severe neurological deficits and limited ambulation. He travelled to the United States in October 2010 where a left IJ vein stent was placed for presumed CCSVI. He was discharged with the recommendation to take daily ASA. In the following weeks, his gait deteriorated and he suffered a significant fall. A postprocedural ultrasound showed thrombus occluding his left IJ vein stent. He was admitted to the Stroke Service at Foothills Medical Centre. Subsequent dedicated CT venography demonstrated reduced flow and possible venous thrombosis extending from the left IJ vein into the sigmoid sinus and transverse sinus (Figure 6a and b). He was treated with anticoagulation and transferred back into the care of his local hospital.

\section{Conclusions}

We have reported five cases representing a range of moderate to severe complications associated with both angioplasty and stent placement in IJ and azygous veins for presumed CCSVI. While some complications are initially asymptomatic, namely asymptomatic IJ vein thrombosis and stent migration into the renal vein, these patients face future risks including the potential need for long-term anticoagulation and the possibility of stent thrombosis in the renal vein.

In 2010, the MS Society of Canada awarded grants for seven observational studies in Canada and the United States to determine the validity of the CCSVI hypothesis and further characterize the nature of venous anatomy in MS patients and controls should anomalies be found. We eagerly await the results of these studies, but still anticipate that some patients will continue to seek out CCSVI treatments abroad in the interim.

With respect to the provision of care to patients after they have undergone CCSVI procedures, there is presently no evidence to support radiological monitoring for complications from CCSVI procedures in asymptomatic patients. As well, there are no formal guidelines for management of asymptomatic venous stent thrombosis in IJ vein stents and they should be managed on a case by case basis as deemed appropriate by the appropriate medical professionals. Suspected venous thrombosis involving intracranial, axillary, superior vena cava or pulmonary vessels should be treated as an emergency with appropriate medical therapies and interventions as would be the case in any patient. These recommendations as well as the rationale behind them were recently outlined in a document from the College of Physicians and Surgeons of Alberta (www.cpsa.ab.ca/.../ Managing_patients_with_MS_after_out-of-country_ endovascular_treatment_for_CCSVI.pdf).

Ultimately, whether or not care-providers agree with patients pursuing such treatment, we have to continue to provide care to our patients, be mindful of potential risks, and continue to develop strategies to monitor and manage these patients as their numbers increase. A registry to follow patients who have sought out CCSVI therapy outside of the country is planned in Alberta, while MS caregivers across the country are developing similar strategies. Further experience with these complications and collaboration across Canada and beyond will provide information for the ongoing development of care guidelines for such patients. We caution patients and MS care providers to be aware of the risks associated with these interventions and be vigilant for complications. 


\section{Disclosures}

Dr. Burton is a co-investigator for the Canadian Institutes of Health Research (CIHR) sponsored "Knowledge Synthesis Grant: Evidence On Tap - Systematic reviews of the evidence that tests for chronic cerebrospinal venous insufficiency (CCSVI) are valid and reproducible, that CCSVI is associated with multiple sclerosis, and of the benefits and harms of endovascular therapy for multiple sclerosis". Dr. Hill and Dr. Costello are members of a CIHR advisory panel on CCSVI. Dr. Hill and Dr. Goyal are co-investigators and Dr. Costello is the principal investigator for the MS Society of Canada supported study "Determining the relationship between chronic cerebrospinal venous insufficiency and multiple sclerosis at the University of Calgary.

\section{REFERENCES}

1. Zamboni P, Galeotti R, Menegatti E, et al. A prospective open-label study of endovascular treatment of chronic cerebrospinal venous insufficiency. J Vasc Surg. 2009;50:1348-58.

2. Zamboni P, Galeotti R, Menegatti E, et al. Chronic cerebrospinal venous insufficiency in patients with multiple sclerosis. J Neurol Neurosurg Psychiatry. 2009;80:392-9.

3. Khan O, Filippi M, Freedman M, et al. Chronic cerebrospinal venous insufficiency and multiple sclerosis. Ann Neurol. 2010; 67:286-90.

4. Doepp F, Friedmann P, Valdueza JM, et al. No cerebrocervical venous congestion in patients with multiple sclerosis. Ann Neurol. 2010;68:173-83.

5. Sundstrom $P$, Whalin $A$, Ambarki $K$, et al. Venous and cerebrospinal fluid flow in multiple sclerosis: A case-control study. Ann Neurol. 2010;68:255-9.

6. Wattjes MP, van Oosten BW, de Graaf WL, et al. No association of abnormal cranial venous drainage with multiple sclerosis: a magnetic resonance venography and flow-quantification study. $\mathbf{J}$ Neurol Neurosurg Psychiatry. Epub 2010 Oct 27

7. Baracchini C, Perini P, Calabrese M, Causin F, Rinaldi F, Gallo P. No evidence of chronic cerebrospinal venous insufficiency at multiple sclerosis onset. Ann Neurol. 2011;69(1):90-9.

8. Meyer-Schwickerath $\mathrm{R}$, Haug $\mathrm{C}$, Hacker $\mathrm{A}$, et al. Intracranial venous pressure is normal in patients with multiple sclerosis. Mult Scler. 2011 May;17(5):637-8. Epub 2011 Jan 12.

9. del Pilar Cortes Nino M, Tampieri D, Melancon D. Endovascular venous procedures for multiple sclerosis. Mult Scler. 2010;16(7):771-2.

10. Ludyga T, Kazibudzki M, Simka M, et al. Endovascular treatment for chronic cerebrospinal venous insufficiency: is the procedure safe? Phlebology. 2010;25(6): 286-95.

11. Samson K. Experimental multiple Sclerosis vascular shunting procedure halted at Stanford. Ann Neurol. 2010;67(1):A13-15. 